Recherches en histoire de l'art, histoire des civilisations, archéologie, anthropologie et muséologie

\title{
Exposer la sculpture au Louvre au temps de Rodin, 1860-1914
}

\section{Geneviève Bresc-Bautier}

\section{OpenEdition}

Journals

Édition électronique

URL : http://journals.openedition.org/cel/337

DOI : $10.4000 /$ cel.337

ISSN : 2262-208X

Éditeur

École du Louvre

\section{Référence électronique}

Geneviève Bresc-Bautier, «Exposer la sculpture au Louvre au temps de Rodin, 1860-1914 », Les Cahiers de l'École du Louvre [En ligne], 8| 2016, mis en ligne le 01 mai 2016, consulté le 17 septembre 2019. URL : http://journals.openedition.org/cel/337 ; DOI : 10.4000/cel.337

\section{(2) $(\mathcal{Q} \Theta \Theta$}

Les Cahiers de l'École du Louvre sont mis à disposition selon les termes de la licence Creative Commons Attribution - Pas d'Utilisation Commerciale - Pas de Modification 4.0 International. 


\title{
Cahiers de l'École du Louvre
} recherches en histoire de l'art, histoire des civilisations archéologie, anthropologie et muséologie

\section{Numéro 8. Mai 2016}

\author{
Exposer la sculpture au Louvre
} au temps de Rodin, 1860-1914. Geneviève Bresc-Bautier

Article disponible en ligne à l'adresse :

http://www.ecoledulouvre.fr/cahiers-de-l-ecole-du-louvre/numero8-mai2016/Bresc-Bautier.pdf

Pour citer cet article :

Geneviève Bresc-Bautier, « Exposer la sculpture au Louvre au temps de Rodin, 18601914.», Cahiers de I'École du Louvre. Recherches en histoire de I'art, histoire des civilisations, archéologie, anthropologie et muséologie [en ligne] n 8 , mai 2016, p. 29 à 40 .

\section{@creative \\ ㅇ.. $\odot \circledast$}

(c) École du Louvre

Cet article est mis à disposition selon les termes de la Licence Creative Commons Attribution - Pas d'utilisation commerciale - Pas de modification 3.0 non transposé. 


\section{Cahiers de l'École du Louvre}

recherches en histoire de l'art, histoire des civilisations archéologie, anthropologie et muséologie

\section{Numéro 8. Mai 2016}

\section{Sommaire}

\section{Éditorial}

\section{Articles}

Malcolm Baker, Distinguished Professor of the History of Art, University of California, Riverside Writing about displays of sculpture: historiography and some current questions....

Catherine Chevillot, Directrice du Musée Rodin

Les enjeux d'une rénovation.

Dominique Brard, Architecte

Le Musée Rodin de 2015 : choix muséographiques

p. $20-28$

Geneviève Bresc-Bautier, Conservatrice générale du patrimoine honoraire Exposer la sculpture au Louvre au temps de Rodin, 1860-1914 p. $29-40$

Astrid Nielsen, Conservatrice des collections de sculpture, Albertinum, Dresde Exposer la sculpture dans l'Albertinum de Dresde : passé, présent et futur.....p. 41-50

Colin Lemoine, Historien de l'art, responsable des sculptures au musée Bourdelle

Amélie Simier, Conservatrice en chef du patrimoine, directrice des musées Bourdelle et Zadkine

La sculpture sur le bout des doigts. Retour sur l'élaboration

d'une salle pédagogique et tactile au musée Bourdelle.....

Cecilia Hurley-Griener, Docteur en histoire de l'art (HDR), équipe de recherche, École du Louvre

Putting sculpture on show/Exposer la sculpture : conclusion. p. $58-65$ 


\section{Geneviève Bresc-Bautier}

Exposer la sculpture dans les collections permanentes d'un musée, c'est non seulement la montrer, mais démontrer la beauté, la spécificité, l'évolution d'un art, de ses créateurs et de ses styles. La difficulté de l'exercice est qu'il est destiné à tous, aux érudits et au grand public, aux spécialistes et aux enfants des écoles ; et il faut bien reconnaitre que les conservateurs du XIX ${ }^{e}$ siècle, $s^{\prime}$ ils avaient une certaine idée de la pédagogie, s'adressaient surtout à un public d'artistes et de connaisseurs, d'antiquaires au sens ancien du terme. Leur démonstration, comme de nos jours, était obligatoirement personnelle, inscrite dans leur temps, donc nécessairement vite démodée et critiquable. Ceci explique les incessants remaniements, causés par l'insertion d'œuvres mises au goût du jour, celles du Moyen Âge en particulier.

\section{Des contraintes multiples : I'espace, la lumière, le choix des œuvres}

Un musée permanent n'est ni un livre, ni une exposition temporaire, mais subit des contraintes imposées. Elles dépendent de différents facteurs. Le premier est constitué par la collection elle-même, qui a été réunie dans des circonstances historiques variées et qui s'étoffe à la fois en fonction des occasions et d'une politique volontariste. La liste d'œuvres destinées aux salles est fluctuante, selon que l'on choisit d'exposer ou de laisser en réserve. Ce choix est dicté par une appréciation subjective, parfois en raison de l'état physique, ou du jugement critique, ou du peu de relation avec les autres ouvres exposées. En outre, la collection est en perpétuelle augmentation, les achats étaient relativement limités par la pénurie financière, mais les dons étaient nombreux. De plus, tous les conservateurs de l'époque - et surtout Louis Courajod - ont mené une politique volontariste de regroupements, d'échanges, de transmissions et de mises en dépôt qui a profité à toutes les époques, surtout au Moyen Âge et au XviI ${ }^{\mathrm{e}}$ siècle, alors que le musée du Luxembourg envoyait les sculptures des artistes décédés.

Ces sculptures ne se présentent pas de façon uniforme, selon leur dimension, leur forme, leur poids, leur matière, le point de vue privilégié qui découle de leur fonction première. Il faut donc choisir un mode de présentation : socle pour les ronde-bosse et cimaise pour les reliefs.

L'espace est une contrainte majeure dans le Louvre palatial. Depuis longtemps, on réservait aux sculptures des espaces en rez-de-chaussée, afin de limiter l'effet de leurs poids. Les conservateurs ont dû bon gré mal gré composer avec les salles existantes, de dimensions diverses, généralement de hauteur sous plafond importante. Comme le lit de Procuste, il fallait faire entrer de force un sujet de la démonstration (un style, une époque) dans le carcan imposé. Certes au XIX ${ }^{\mathrm{e}}$ siècle, les normes de circulation et de sécurité n'étaient pas en vigueur, et il ne fallait pas $1,40 \mathrm{~m}$ pour un passage. Une dernière contrainte enfin était constituée par les faibles moyens du musée et par l'autorité de l'architecte du palais.

La petite "galerie d'Angoulême " inaugurée en 1824, s'était vite révélée trop exiguë, à mesure que les acquisitions se multipliaient. Il a donc fallu chercher de nouveaux espaces qui dépendaient de la structure architecturale préexistante ; grande salle ou rabicoin aveugle, couloir pompeusement dénommé vestibule, ou cagibi promu "cabinet». Bien entendu, la surface n'en était jamais modulable, et il fallait s'adapter au rythme conçu pour d'autres besoins par des architectes classiques, morts depuis longtemps. Une autre contrainte découlait de la lumière, heureusement naturelle avant l'invention de l'éclairage artificiel, qui n'était évidemment jamais zénithale. Elle provenait de grandes fenêtres, rarement disposées de deux côtés. Si une lumière latérale, qui caresse les reliefs et les fait vibrer, est assez bonne, elle devait être très limitée et laissait dans la pénombre 
la plus grande partie d'une salle. La mise en valeur des œuvres dépendait donc autant de la lumière naturelle que de leur disposition dans la salle.

À cette époque aussi, les contraintes environnementales n'étaient pas observées. Quant au public, peu nombreux et respectueux, il n'avait pas besoin d'être tenu à distance. Il est fort probable cependant que les conservateurs cherchaient à protéger les sculptures en ménageant de larges espaces alentours, ne serait-ce que pour en montrer l'effet de loin.

\section{Une muséographie de la symétrie}

Mettre en valeur et mettre en relation. Le rapport des œuvres entre elles était à la base de leur présentation. Donner au visiteur au premier coup d'œil une vision claire et belle, pour le transformer en spectateur attentif. Tout l'effort de présentation était dirigé sur le regard. Ne pas écraser de petites sculptures par la présence d'immenses marbres; ne pas multiplier les reliefs de dimensions discordantes sur un même mur ; organiser en revanche des rapports d'échelle entre des éléments complémentaires par leur style, ou leur iconographie, ou leur technique. Le travail du conservateur était un peu celui d'un étalagiste, car au XIx ${ }^{e}$ siècle, il n'était pas soumis à un muséographe. L'architecte du Louvre s'occupait du bâtiment, de son décor, des murs, des sols et parfois dessinait des socles. Mais la responsabilité incombait principalement au conservateur, aidé par les ateliers, les marbriers et les menuisiers. Il présidait au choix du circuit, pour lequel il privilégiait la chronologie, le style, les personnalités, ou les trois plus ou moins ensemble.

Trois maitres mots dominaient une muséographie parfaitement ordonnée: centralité, symétrie, unité dans la diversité. Au centre des grandes salles, s'alignaient en général une œuvre pivot, et de part et d'autre deux ou quatre autres, selon leurs dimensions, formant comme la spina d'un cirque antique. Évidemment, la hauteur des socles régnait, quelle que fut celle de la figure présentée. De part et d'autre de portes centrales, reliefs et statues formaient un balancement harmonieux. Deux murs opposés avaient des compositions qui se répondaient, organisées en vis-à-vis. Un grand mur unique et principal portait sa propre logique, toujours marquée par la symétrie et le rythme entre reliefs et ronde-bosse. La perspective n'était pas oubliée, et quand l'enfilade des salles butait sur un mur, ou quand une porte semblait donner une échappée vers la salle voisine, une œuvre devait constituer un appel.

Les vitrines étaient réservées aux salles d'objets d'art, gérées jusqu'en 1893 dans le même cadre administratif, par les mêmes conservateurs. La partition qui survint à cette date, pour contrer la mauvaise entente entre les deux conservateurs, Louis Courajod et Émile Molinier, se fit précisément sur la différence entre objets de vitrine et sculptures de format supérieur à $50 \mathrm{~cm}$. Du coup, les vitrines pour les petits objets n'apparurent qu'au début du xxe siècle, quand André Michel commença à acquérir des esquisses de terre cuite, tant du $\mathrm{XvIII}^{\mathrm{e}}$ siècle que de Carpeaux, ou des petits groupes animaliers de Barye ${ }^{1}$. Il introduisit aussi un chevalet pour présenter le Scipion du legs Rattier dans la salle Michel-Ange.

Tout ceci supposait une hiérarchie, dictée autant par la qualité intrinsèque et l'importance historique, que par la forme ou par la facilité à mettre en résonance des œuvres symétriques. Le choix du conservateur était alors modelé par sa conception personnelle de l'esthétique des œuvres, par son goût et celui de son temps ainsi que par sa volonté d'organiser l'espace. La notion de chef-d'œuvre s'établit ainsi, privilégiant des pièces centrales, telle la Diane d'Anet, les trois marbres de Puget ou la Fontaine de l'Observatoire de Carpeaux, et rendant plus brouillonnes les salles médiévales, de format plus réduit.

Nous savons peu de chose de la couleur des murs, qui n'a pas toujours été présente. Dans les années 1860, domine la pierre naturelle, aux joints bien visibles et à la belle stéréotomie. Seule la salle Jean Goujon et la salle Jean de Douai

1. Signalées pour les nouvelles acquisitions, dans Paul Joanne, Paris-Diamant, Paris, Hachette, 1905, p. 100, et pour les salles dans Guides Joanne. Le musée du Louvre, Paris, Hachette, 1912, pp. 27-30. 
(ou Michel-Ange) bénéficièrent d'un fond rouge brique de façon à détacher la blancheur des marbres. En 1877, Barbet de Jouy fit peindre en rouge la future salle Rude, la salle Coysevox et la salle Chaudet. Lors des remaniements des années 1880-1890, Courajod et l'architecte Guillaume généralisèrent la couleur.

Les socles et les gaines de marbre essentiellement colorés, étaient de très grande qualité. Le marbre blanc n'était employé que lorsqu'on abordait le néoclassicisme. Une alternance de colonnes rondes et de socles rectangulaires assurait la diversité, même si la hauteur était soigneusement étudiée.

Il est possible de suivre la muséographie des salles par le catalogue publié en 1897 et son supplément de 1907, par les guides de Paris², et aussi par de maigres correspondances ou des interventions des conservateurs au Comité des musées. Les photographies anciennes forment une source incontournable, malheureusement parcellaire, les prises de vue privilégiant toujours les angles les plus photogéniques. Alors que la première photographie d'une salle du Louvre, par Marville, a été tirée en 1855, et que Baldus vint fugacement, c’est dans les années 1863-1865 que la présentation est bien connue grâce aux vues stéréoscopiques d'Henri Plaut, d'Hippolyte Jouvin et d'Alexandre Bertrand. Elles remplacent avantageusement les rares gravures des journaux des années 1857-1858. Par la suite, les photographes (Prosper Deloeul, Édouard Dontenvill) se spécialisèrent dans les vues d'œuvres isolées.

\section{Des conceptions successives}

Le premier musée de sculpture « moderne » au Louvre, la galerie d'Angoulême, ouverte en 1824, était disposé dans cinq salles de l'aile ouest de la cour Carrée, entre le pavillon de l'Horloge et celui de Beauvais ${ }^{3}$. L'architecture était en grande partie celle de Jacques Lemercier, remaniée par Pierre François Léonard Fontaine sous l'Empire.

\section{Léon de Laborde, 1850-1851}

En 1848 et 1851, Adrien de Longpérier et Léon de Laborde avaient séparé la collection en deux entités : au rez-de-chaussée de l'aile méridionale de la cour Carrée, cinq salles pour le Moyen Âge, la Renaissance et le début du XviI ${ }^{\mathrm{e}}$ siècle, et cinq autres salles dans l'ancienne galerie d'Angoulême, pour les œuvres du XVII $^{\mathrm{e}}$ au début du XIX ${ }^{\mathrm{e}}$ sièclét.

Pour visiter les collections les plus anciennes, le visiteur entrait par la porte centrale de la demie-aile et pénétrait dans un mince vestibule, presque un couloir, où avaient été disposées les premières sculptures médiévales entrées au Louvre: trois gisants, la Vierge normande de Blanchelande et le beau Childebert de SaintGermain-des-Prés 5 . Puis, il abordait perpendiculairement une enfilade de salles, de format différent selon les contraintes des murs porteurs, éclairées par des grandes fenêtres au Sud. La présentation n'était pas conçue de façon strictement chronologique, afin de laisser les beaux espaces aux collections les plus appréciées. Ainsi, après le vestibule, on pénétrait d'abord dans la grande salle de Jean Goujon, consacrée à la Renaissance française. Ce choix n'avait rien d'anodin puisqu'il

2. En particulier la série des guides d'Adolphe, puis de $\mathrm{P}$. Joanne, $1863,1867,1873,1874$, $1878,1879,1881,1882,1883,1887,1889,1890,1893,1895,1898,1905,1912$.

3. Sur les salles de I'aile sud, Christiane Aulanier, Le musée Charles $X$ et le département des Antiquités égyptiennes, Paris, Éditions des Musées nationaux, 1961, pp. 95-99. Sur les salles de l'aile ouest, idem, Le pavillon de l'Horloge et le département des Antiquités orientales, Paris, Éditions des Musées nationaux, 1964, pp. 120-126.

4. Sur la muséographie de Laborde, Sophie Martin, Véronique Milande, en collaboration avec Nathalie Bondil, « Léon de Laborde (1807-1869) : de la galerie d'Angoulême au département des Sculptures », Actes du colloque Un combat pour la sculpture : Louis Courajod (1841-1896), historien et conservateur, Paris, musée du Louvre, 15 janvier 1996, publiés sous la direction de Geneviève Bresc-Bautier avec la collaboration de Michèle Lafabrie, Paris, Rencontres de I'École du Louvre, 2003, pp. 22-27.

5. Elles n'avaient pas grand succès, Joanne en 1867 a l'audace d'écrire : « La sculpture du moyen âge pourra fournir, dès qu'on songera sérieusement à en réunir des échantillons bien choisis, au moyen de moulages, à défaut d'œuvres originales, une division intéressante du musée des Antiques, mais dans l'état actuel, il n'y a pour ainsi dire que le titre », A. Joanne, Paris : nouveau guide de l'étranger et du parisien, Paris, Hachette, 1867, p. 670. 
consacrait le sculpteur le plus aimé, le "Praxitèle français ». Au centre, trônait la Diane d'Anet, et dans l'axe longitudinal, deux groupes en symétrie l'encadraient : les Trois Grâces du monument du cour d'Henri II, faisant pendant à quatre cariatides de bois de la châsse de Sainte Geneviève, posées sur un socle copié sur celui des Grâces. Deux chiens en bronze, de Pierre Ir Biard, que l'on croyait provenir de la fontaine d'Anet, étaient placés près de la Diane. Sur le grand mur, la cheminée de Villeroy occupait le centre, entre deux colonnes funéraires, celles d'Anne de Montmorency et celle des Cossé (pourtant du XviI ${ }^{\mathrm{e}}$ siècle). Aux murs, petits et grands reliefs de Goujon côtoyaient les groupes funéraires de Pilon (le priant du cardinal de Birague et les figures de Valentine Balbiani) et attribué à Jean Cousin (Philippe Chabot), ainsi que plusieurs bustes, avec également quelques sculptures régionales, de Ligier Richier ou attribuée à Pierre Jacques, de Reims, qui montraient clairement la volonté de Laborde de présenter l'ensemble du territoire national, sans se limiter au prestigieux art royal.

Puis, pour suivre le circuit, il fallait tourner soit à droite, soit à gauche. Vers la droite, on entrait la salle de Jean de Douai c'est-à-dire de Jean Bologne, qui mettait l'accent sur un artiste considéré comme "national ", auteur du Mercure, et (croyait-on à tort) de l'Enlèvement de Psyché, un bronze d'Adrien de Vries. Laborde l'avait fait venir de Saint-Cloud pour le mettre en bonne place, au centre. Pourtant la salle montrait surtout les grands chefs-d'œuvre de la sculpture italienne, les Esclaves de Michel-Ange, les premières œuvres attribuées aux Della Robbia acquises en 1847 et 1851, les reliefs de Riccio et la Nymphe de Fontainebleau par Cellini, mais aussi divers reliefs de la Renaissance germanique, nouvellement acquis. Les Esclaves étaient placés en vis-à-vis, à côté des portes, afin de recevoir une bonne lumière du Sud.

Dans la suite, vers l'extrémité de l'enfilade, une petite salle de Michel Colombe, consacrée à la première Renaissance, abritait le tombeau des Ponchier et celui de Commynes, venu de Versailles, entourant le retable de Gaillon, qui formait le point focal de la perspective de l'enfilade sur la Seine.

En retournant vers la salle Jean Goujon, qu'il fallait retraverser, on accédait à la salle des Anguier, plus petite, où pourtant s'entassaient des sculptures funéraires, dont au centre, la Pyramide des Longueville des Anguier. Un mur était occupé par l'ensemble du monument du Pont-au-Change de Simon Guillain (venu de Versailles) devant lequel se détachait la Renommée qu'on ne savait pas encore être de Pierre Ir Biard.

Derrière encore s'ouvrait une petite salle de Francheville (Pierre Francqueville), pour exposer les captifs en bronze de la statue d'Henri IV au Pont-Neuf.

Laborde avait aussi installé un petit musée de moulages de sculptures médiévales et Renaissance dans l'angle sud-est de la cour Carrée, à proximité des salles de sculptures de la Renaissance. On y voyait la copie en plâtre de la cheminée du Franc de Bruges, chef-d'œuvre flamboyant exécuté pour le palais communal de Bruges par Lancelot Blondel, en 1528-1531, entourée des moulages des tombeaux de Charles le Téméraire, de Marie de Bourgogne et de deux rois espagnols.

Du côté nord-ouest de la cour, dans l'ancienne galerie d'Angoulême, qu'on abordait par la porte située au centre de la façade de l'aile Louis XIII, Laborde remania complétement les cinq salles en enfilade auxquelles il attribua le nom de cinq sculpteurs : Coysevox, Puget, les Coustou, Bouchardon et Houdon. Dans une évolution globale qui allait du XVII ${ }^{e}$ au XIx ${ }^{e}$ siècle, Laborde voulait manifester la qualité des personnalités artistiques majeures De même qu’on pénétrait d'abord au Sud dans la grande salle de Jean Goujon, ici c'était Puget qui avait l'honneur d'une vaste pièce d'entrée. Laborde y avait rassemblé ses chefs-d'œuvre : Milon de Crotone, l'Hercule gaulois (au centre) et Persée et Andromède (spécialement venu de Versailles) formaient l'alignement central, posés sur de nouveaux socles en marbre vert de mer sur base de noir portor. À gauche, la salle de Coysevox plus petite, accueillait les bustes du grand portraitiste et, sur le mur du fond, le grand tombeau de Mazarin. Il fallait retraverser la salle des Puget pour aborder la salle des Coustou, petite et surpeuplée, qui abritait alors les reliefs de la place des Victoires de Desjardins, à côté des réalisations des deux frères. Dans la salle de 
Bouchardon, à la belle architecture qui avait gardé la forme de la chapelle du $\mathrm{XvII}^{\mathrm{e}}$ siècle, la Psyché abandonnée de Pajou avait aussi sa place. Le circuit s'achevait par la salle de Houdon au rez-de-chaussée du pavillon de Beauvais, largement éclairée par trois fenêtres sur la rue de Rivoli. C'est ici qu'étaient exposés les marbres de Canova.

L'ordre chronologique des œuvres n'était pas toujours exactement respecté. Ainsi, la statue de Louis XIV par Coysevox et celle de Louis XIII par Coustou appartenant au même ensemble du Vơu de Louis XIII, exécuté pour le chœur de Notre-Dame de Paris, étaient situées à deux salles d'écart pour bien affirmer la primauté de la présentation esthétique sur l'historique, et aussi pour s'opposer aux revendications de l'archevêque de Paris, qui eut finalement gain de cause, récupérant les statues en 1866. Leur position centrale exprimait leur importance.

\section{Henry Barbet de Jouy (1863-1879)}

Après le départ de Laborde, Henry Barbet de Jouy transforma la présentation ${ }^{6}$. Il avait préparé dès 1859 un "futur musée chrétien ", avec une mosaïque et le sarcophage de l'évêque de Soissons, saint Drausin (vi siècle), qu'il ouvrit en novembre 1868, du côté nord des salles de la Renaissance, vers la cour Carrée. Il remania aussi les autres salles de l'aile sud de la cour Carrée, ouvertes en mars 1868.

L'acquisition de la collection Campana en 1863, puis le sauvetage des sculptures de Saint-Cloud, de Compiègne et des Tuileries, dont Barbet de Jouy avait été l'artisan en 1870 et 1871, apportèrent de nouvelles recrues en nombre.
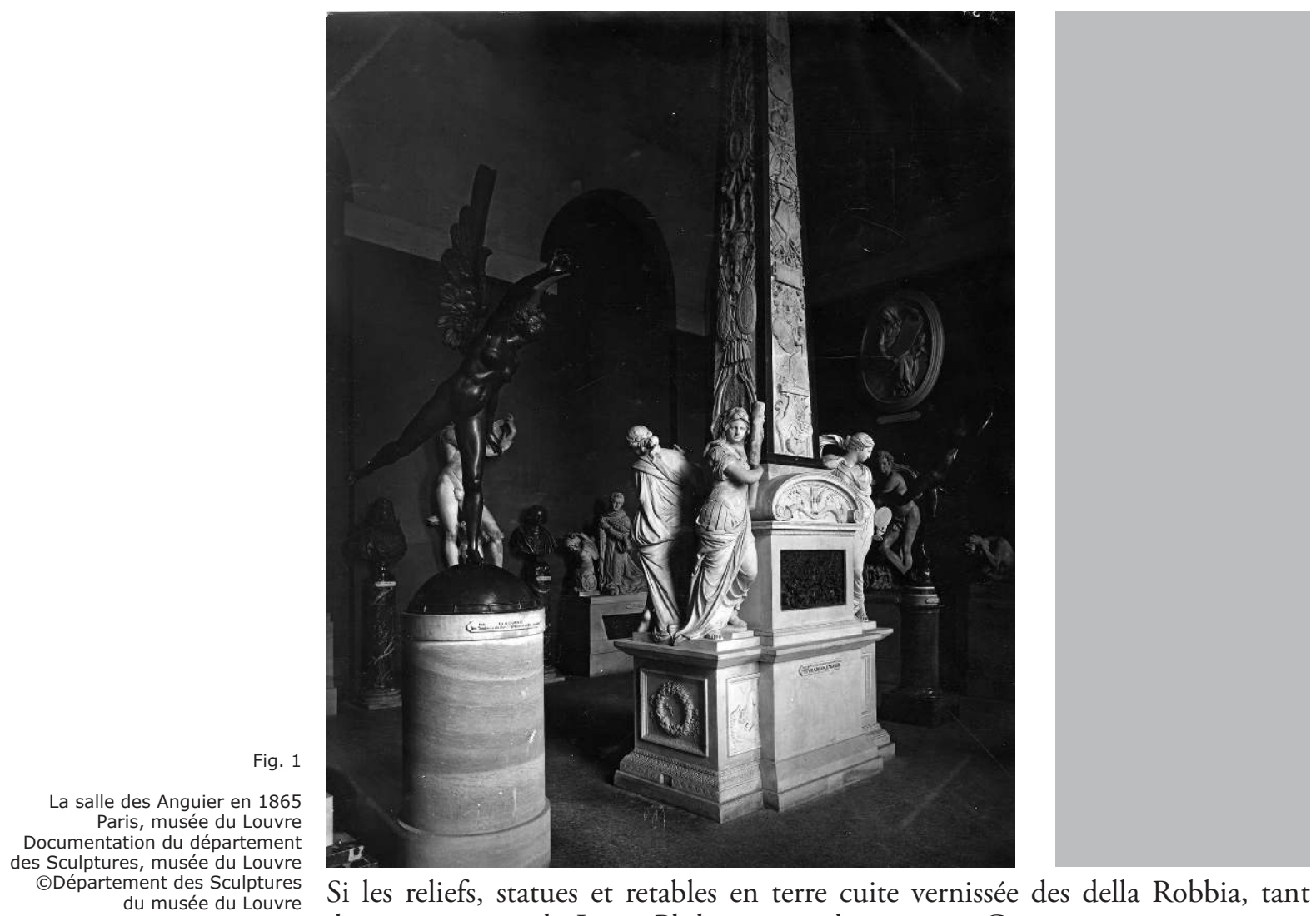

Si les reliefs, statues et retables en terre cuite vernissée des della Robbia, tant des acquisitions de Louis-Philippe que du marquis Campana, rejoignirent un nouveau "musée de la Renaissance " ouvert en 1863 dans l'aile nord de la cour Carrée, à l'étage, ce qui allégeait la salle de Jean de Douai, il restait des marbres

6. Sur la muséographie de Barbet de Jouy, Isabelle Gaétan, en collaboration avec Emmanuelle Héran, « Henry Barbet de Jouy (1812-1896), de la bonne gestion des collections », Actes du colloque Un combat pour la sculpture : Louis Courajod (1841-1896), historien et conservateur, Paris, musée du Louvre, 15 janvier 1996, publiés sous la direction de G. Bresc-Bautier avec la collaboration de Michèle Lafabrie, Paris, Rencontres de I'Ecole du Louvre, 2003, pp. 44-47. Les salles sont décrites dans le guide Joanne : A. Joanne, Paris : nouveau guide de l'étranger et du parisien, Paris, 1867, pp. 670-672. 
à caser. La salle de la cheminée de Bruges, pourtant remaniée en 1868, fit office de réserve aménagée, une sorte de capharnaüm surpeuplé. Deux autres chiens de bronze, venus de Saint-Cloud en 1870, vinrent encadrer la Diane d'Anet. Le Mercure de Jean Bologne émigra dans la salle des Anguier, pour faire pendant à la Renommée (de Biard) (fig. 1), si bien que la salle Jean de Douai devint très normalement salle de Michel-Ange, d'où avait été évacuée une œuvre considérée comme " française ». Elle reçut pour former le centre du mur principal, ApollonJason sauvé du parc de Saint-Cloud.

Mais l'acquisition de l'immense porte du palais Stanga de Crémone bouleversa cette salle Michel-Ange. Pour la placer, au centre du mur ouest, comme le proposait l'architecte du Louvre, Hector Lefuel, il fallait supprimer la longue enfilade qui était auparavant décentrée vers la Seine, pour créer le point fort d'une nouvelle perspective. La porte fut encadrée par les Esclaves de Michel-Ange en pendant, laissant apercevoir la petite salle suivante, celle de Michel Colombe. Dans l'ouverture s'encadrait la Vierge d'Olivet, nouvellement acquise. Au milieu de la pièce, la fontaine circulaire, censée provenir du château de Gaillon, formait le pivot d'une présentation assez touffue, où s'étageaient des reliefs au-dessus de bustes ou de statues. Elle en avait chassé l'Enlèvement de Psyché, condamné à perdre sa patine dans le jardin des Tuileries. Sur l'autre mur, la Nymphe de Fontainebleau, jusqu'alors posée sur un socle assez bas, fut mise dans le tympan d'une arcade, dans une position en rapport avec sa destination primitive (fig. 2). Cette innovation considérable, inaugurée en $1876^{7}$, eut un grand succès.
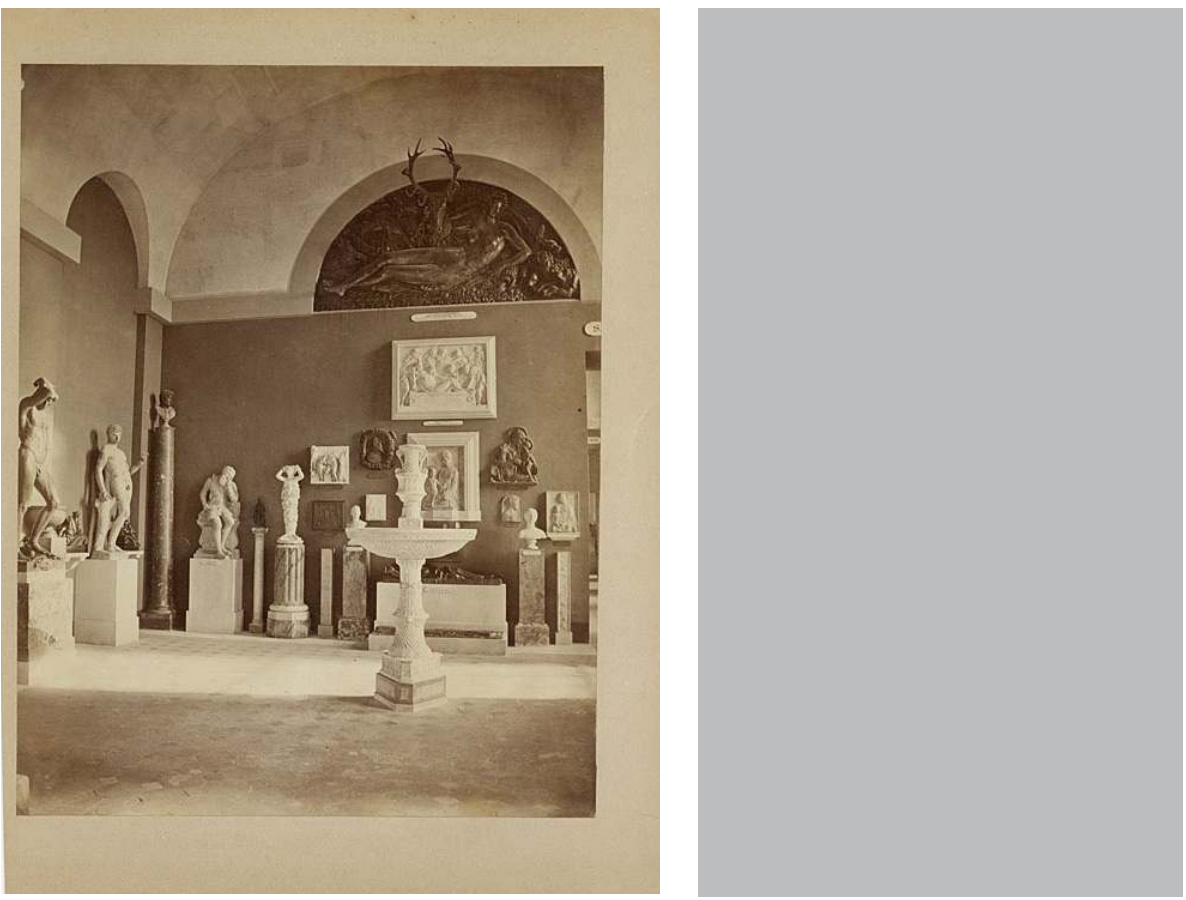

Les salles modernes furent aussi remodelées. Si le Vou de Louis XIII repartit à Notre-Dame, les salles du XVII et du XVIII ${ }^{e}$ siècle se remplirent de petits groupes de morceaux de réception, venus de l'École des beaux-arts, et des grands marbres des Tuileries (Chasseur et César de Nicolas Coustou, Fanne de Coysevox, Annibal de Slodtz). Dans la salle de Coysevox, le Faune flutteur, et dans la salle des Coustou, le Chasseur, occupèrent respectivement la place centrale laissée par Louis XIV en prière et Louis XIII offrant sa couronne des mêmes auteurs. La salle Puget fut transformée par l'adjonction des quatre termes venus de Saint-Cloud, adossés aux piliers centraux, ainsi que de grands moulages des Atlantes de Toulon, hommage appuyé au sculpteur provençal, considéré par Michelet et les Romantiques comme un génie incompris au temps de l'absolutisme, sur lequel Léon Lagrange avait publié une première et célèbre monographie en 1868 . 
Alors que la salle des Coustou accueillait pêle-mêle Bouchardon, Allegrain, Pigalle et Falconet, Barbet de Jouy transplanta Houdon dans la salle de Bouchardon, qui perdit son nom, au profit du nouveau venu, La Diane de bronze de Houdon avait en effet été disposée au centre, sur un socle circulaire qui s'adaptait parfaitement à l'étoile de marbre du luxueux pavement. En revanche, les travaux du Nouveau Louvre de Napoléon III firent disparaître l'éclairage par les fenêtres de l'ouest, et il fallut mettre en pleine lumière dans la seule ouverture restante le grand groupe de L'Amour et Psyché par Delaistre.

En corollaire, en 1863, l'ancienne salle de Houdon devint celle de Chaudet, garnie des nouveaux envois du musée du Luxembourg. Le Louvre s'ouvrait aux grands contemporains décédés depuis peu: Pradier (mort en 1852), David d'Angers (mort en 1856), Rude (mort en 1855). Cette très grande salle occupait tout l'angle nord-ouest, au rez-de-chaussée du pavillon de Beauvais, éclairée au Nord par trois grandes fenêtres. La ligne centrale était composée de cinq grandes pièces alignées face aux fenêtres, tournant le dos au visiteur qui entrait. Au centre, le grand bronze de Rude, Mercure attachant sa talonnière, sur un socle rond ; de chaque côté, une figure de Pradier de forme rectangulaire, Atalante et le Niobide blessé; auxquels succédaient aux extrémités, Edipe et Phorbas de Chaudet et Aristée de Bosio. Les parois étaient formées de grandes arcades rythmées de pilastres ; au centre de chacune un grand marbre, encadré soit de deux bustes, soit de deux statuettes (Homère de Roland, Daphnis et Chloé de Cortot, Nisus et Euryale de Roman, Philipæemen de David d'Angers...).

Puis l'agrandissement se prolongea dans l'aile nord grâce au départ du général Lepic, gouverneur du palais, donc la fonction était supprimée. Mais les travaux furent longs et ce n'est qu'en décembre 1877 que fut ouverte la salle Rude, peinte de rouge, décorée d'une frise au pochoir à la base du plafond. Elle était conçue comme le lieu du romantisme, qui intégrait David d'Angers, Ramey et Pradier.

La salle Rude avait privé la salle de Chaudet du Mercure qui en était le centre, mais aussi avait entraîné le départ d'une grande partie de l'art des années 1830. Il fallut donc la réorganiser et la peupler. Désormais, ce fut L'Amour et Psyché de Canova qui forma le groupe central. D'un mur à l'autre, pas moins de sept socles tous rectangulaires formaient la spina centrale face aux fenêtres. Sur chaque pilastre du pourtour, une statue; dans chaque arcade, trois œuvres. Le moindre recoin était habité, mais toujours avec symétrie : pour une statue deux bustes ou deux statuettes en garniture. L'idée était de privilégier le néoclassicisme, en y transférant des morceaux de réception les plus récents (Deseine, Julien), précédemment exposés dans la salle Houdon, dont la présentation fut légèrement allégée.

\section{Louis Courajod (1879-1896): le temps de l'accumulation}

Sous l'autorité nominale d'Edmond Saglio, l'action de Louis Courajod fut déterminante dans le redéploiement complet des salles bien qu'il ne dirigeât le département constitué de façon indépendante, que de 1893 à sa mort, trois ans plus $\operatorname{tard}^{8}$. Mais en tant que conservateur-adjoint depuis 1874, c'est lui qui présentait les acquisitions au comité des conservateurs et proposa son programme d'agrandissement en $1883^{\circ}$. Ses projets muséographiques s'inscrivaient dans sa recherche scientifique sur le Moyen Âge, la Renaissance française et le Quattrocento, qui allait de pair avec de nombreuses acquisitions. Avec passion, il rechercha des œuvres qui avaient été présentées au musée des Monuments français d'Alexandre Lenoir, dont le Louvre était selon lui (et selon Laborde déjà) l'héritier légitime.

8. Dominique Vin, « Louis Courajod et la muséologie du département des Sculptures du musée du Louvre (1874-1896) », Actes du colloque Un combat pour la sculpture : Louis Courajod (1841-1896), historien et conservateur, Paris, musée du Louvre, 15 janvier 1996, publiés sous la direction de G. Bresc-Bautier avec la collaboration de Michèle Lafabrie, Paris, Rencontres de l'École du Louvre, 2003, pp. 59-89.

9. Louis Courajod, « Le futur musée de la sculpture du moyen âge, de la Renaissance et des Temps modernes », Chronique des arts et de la curiosités, 1884, pp. 229-231, 426-428, 440 442 , 448-450 (communication à la société des antiquaires de France, 1883). Ce programme n'est qu'une liste des œuvres du musée des monuments français récupérées ou identifiées. 
Sa première action fut dirigée vers la constitution de vraies salles médiévales au Sud, grâce au départ des ateliers de marbrerie et de menuiserie, et au transfert de la Première Renaissance dans une salle plus grande. À partir de 1881, l'architecte Edmond Guillaume assura les travaux, alors que Courajod sélectionnait dans les "chantiers de Saint-Denis ", tout un ensemble de sculptures qui avaient été portées dans l'abbatiale par Lenoir et que les restaurations drastiques de Violletle-Duc avaient rendues inutilisées. Mais les ouvertures définitives furent retardées à la décennie suivante par l'appétit de Courajod à se procurer des "spécimens " représentatifs, tant à Saint-Denis, que dans les dépôts des cathédrales et des églises ${ }^{10}$, qu'à Versailles ou à l'École des beaux-arts.

Deux nouveaux espaces furent consacrés au Moyen Âge : l'ancienne petite salle Francheville devint la "salle gothique » et l'ancien atelier de marbrerie la «salle André Beauneveu ", située sous l'escalier méridional de la Colonnade. Dans la première, Courajod inséra vers le Nord une porte du palais Sorell de Valence, acquise en 1883. Cette idée, qui imitait la disposition de la porte Stanga, sera reprise dans les années 1930 par Paul Vitry qui systématiquement disposera des portails dans les nouvelles salles afin de donner un contexte architectural. Elle correspondait au goût des grands collectionneurs pour les évocations d'ambiance médiévale. Comme dans la salle Michel-Ange, mais en proportions réduites, Courajod encadra la porte de Valence d'une Vierge gothique et du Childebert de Saint-Germain-des-Prés. Pour contextualiser ces œuvres, il les dressa sur des colonnettes à chapiteaux et les surmonta de dais provenant de tombeaux. De très nombreux fragments architecturaux, récoltés sur les chantiers des cathédrales et des églises, montaient à l'assaut des murs.

L'ambiance recueillie de la salle André Beauneveu était funéraire. L'espace sombre, bas de plafond, montrait au centre une ligne de gisants allongés les pieds vers l'Est (dont celui de Charles V, considéré Philippe VI par Beauneveu), qui débouchait sur le tombeau de Philippe Pot, acquis en 1889, éclairé par un soupirail. Ici aussi, la surpopulation menaçait les parois.

À proximité, la salle des Anguier ferma en 1883, pour devenir la salle de Michel Colombe, dont le contenu provenait de l'ancienne petite salle de ce nom. Ainsi le grand sculpteur disposait-il d'un espace un peu moins étroit que précédemment, où Courajod, grand spécialiste de la première Renaissance française, pouvait mettre en valeur ses trouvailles. Avide et passionné, il y accumula tout ce qu'il pouvait rassembler comme œuvres "franco-italiennes", sur plusieurs rangs. Curieusement il disposa au centre un alignement de trois bronzes maniéristes sur des socles colonnaires : L'Enlèvement de Psyché par De Vries au centre (puisque plus grand), revenu des Tuileries en $1889^{11}$, entre le Mercure de Jean Bologne et la Renommée. Un groupement qui avait peu de rapport avec les gisants et les œuvres scellées sur les parois

La salle principale, toujours dédiée à Jean Goujon, changea peu, même si Courajod exclut les Chiens de Biard, désormais reconnus par lui comme d'époque Henri IV et envoyés cantonner la Diane de bronze de Prieur dans le vestibule d'entrée vers 1896. Il y inséra quelques nouveautés, telle la Vierge de douleur de Pilon.

La salle Michel-Ange reçut peu à peu la moisson de sculptures florentines ou lombardes que Courajod parvenait à faire entrer. La comparaison d'un même mur à dix ans de distance montre une accumulation de reliefs, sur trois, voir quatre rangs (fig. 2 et 3 ). Mais le conservateur était toujours soucieux de régularité : les bustes formaient des pendants, les reliefs étaient de dimensions dégressives. Si des Vierges florentines étaient juchées au-dessus des Esclaves de Michel-Ange, elles étaient organisées en exacte symétrie.

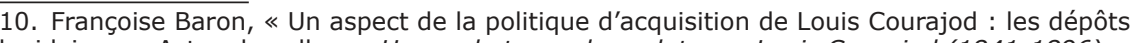
lapidaires », Actes du colloque Un combat pour la sculpture : Louis Courajod (1841-1896), historien et conservateur, Paris, musée du Louvre, 15 janvier 1996, publiés sous la direction de G. Bresc-Bautier avec la collaboration de Michèle Lafabrie, Paris, Rencontres de l'École du Louvre, 2003, pp. 107-124.

11. Chronique des Arts, 20 mai 1889, p. 165 ; 3 août 1889, p. 212. 

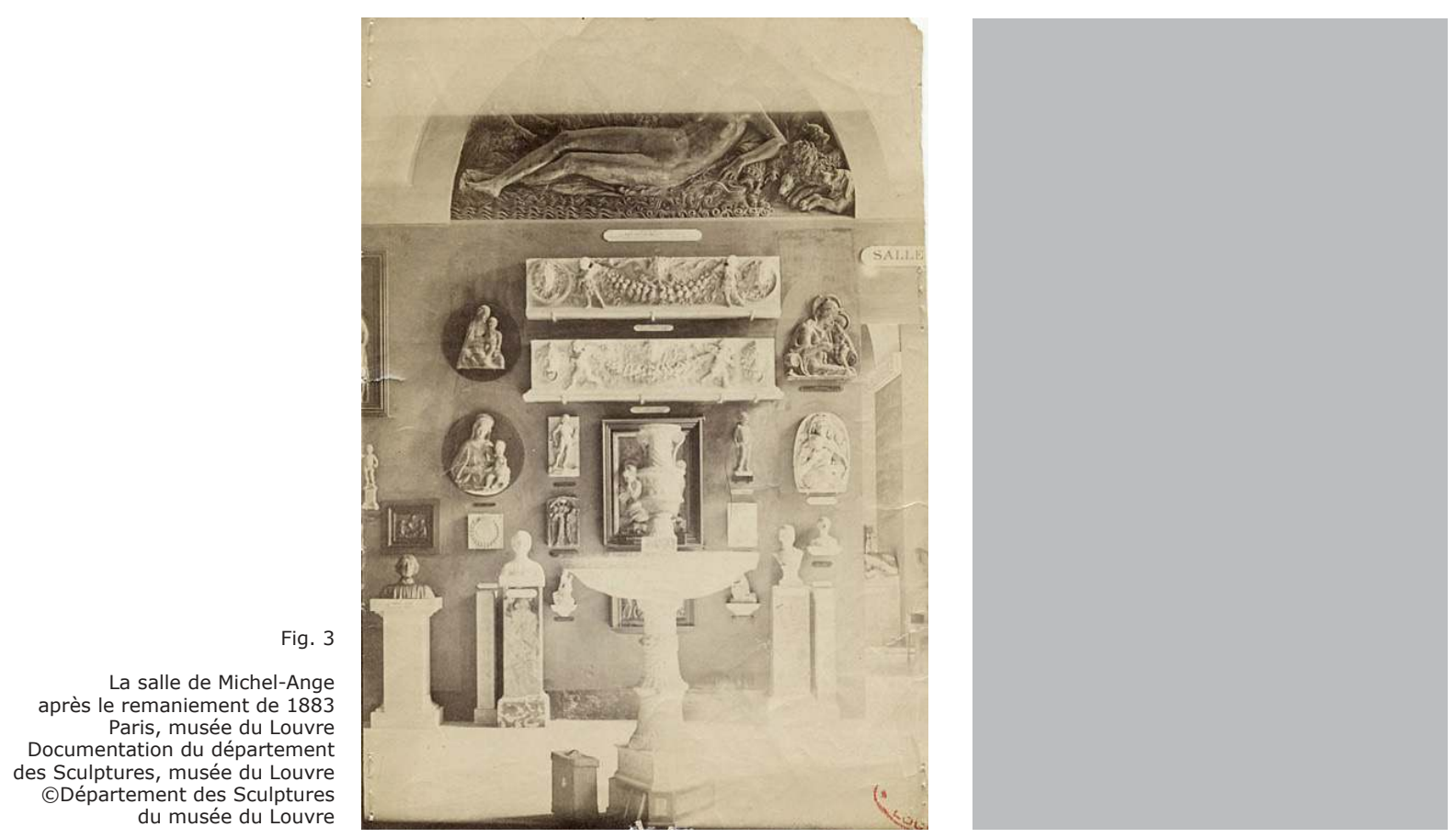

Dans la suite de la salle Michel-Ange, la salle de Michel Colombe devint la petite salle italienne, ou salle de la Renaissance, très étroite. Fait rare, elle n'avait plus de nom emblématique, jusqu'à ce que celui de Donatello s'imposât. Elle développait un panorama de l'art italien du haut Moyen Âge à la Renaissance. Peu peuplée à l'origine, elle connut la même tendance à l'accumulation que la salle voisine, à mesure qu'entraient les dons ou les acquisitions dont ceux qui provenaient des grands amateurs, Timbal ou Davillier. Du coup, la perspective depuis la salle Michel-Ange devint brouillonne, jusqu'à ce que l'acquisition de la grande Vierge de Jacopo della Quercia (1896), trois mois avant la mort de Courajod, et l'action plus calme de son successeur, André Michel, permit de mettre ce grand relief dans l'axe.

Une nouvelle salle s'ouvrit au Nord de cette petite salle italienne, la salle des Della Robbia, à la place des Antiquités judaïques. Courajod n'eut pas le temps de la terminer et André Michel l'ouvrit en $1897^{12}$, avec les terres cuites robbiesques qui étaient revenues du "musée de la Renaissance ». Ici encore une muséographie de l'accumulation (symétrique certes), étouffante par l'abondance des reliefs scellés aux murs jusqu'aux voûtes, ne permit pas à André Michel de mettre en valeur le beau relief de la Madone d'Auviller par Agostino di Duccio, en 1903, alors qu'au centre le Saint Christophe de Francesco di Giorgio Martini (attribué alors à Vecchietta) honorait non seulement l'art siennois, mais son donateur, Eugène Piot (1890).

Dans le petit jeu de taquin, qui transportait les œuvres de salle en salle, Courajod n'eut pas le temps de s'intéresser aux deux espaces situés au nord des nouvelles salles médiévales ${ }^{13}$. La salle de la Cheminée de Bruges reçut un temps le contenu de la salle des Anguier, entre 1883 et 1893, mais elle n'avait pas encore de sens précis.

Courajod procéda aussi à une refonte complète des salles de sculpture moderne. La salle Coysevox, remaniée en 1894, s'enrichit de la pyramide des Longueville d'Anguier, revenue de l'aile sud, et qui put servir de pivot central, devant les statues priantes du XvII ${ }^{\mathrm{e}}$ siècle. Mais il fallut aussi y caser les œuvres de jardin, la Nymphe à la coquille de Coysevox ou la Compagne de Diane de Frémin, échappées aux intempéries de Versailles ${ }^{14}$, quoique assez encombrantes.

12. Elle figure dans P. Joanne, Paris-diamant, Paris, Hachette, 1895, pp. 114-115, comme non installée, et sur le plan du guide Joanne de 1896 ,

13. Deux salles citées comme fermées ibid. dans P. Joanne, op. cit. note 12

14. Respectivement en 1890 et en 1884. 
La grande salle Puget fut transformée complétement. Si la file des chefsd'œuvre du grand sculpteur provençal fut à peine changée par l'introduction des deux vases de Girardon ${ }^{15}$ dans les interstices entre les grands marbres, les quatre parois d'angle, autour des portes axiales, devaient offrir de grands monuments. Les Atlantes de Puget furent envoyés en 1893 au musée de sculpture comparée, avec la Cheminée de Bruges, car pour Courajod, Puget était comme Poussin de "faux parrains, de faux patrons... de l'école nationale ». Les quatre beaux murs, qui encadraient les portes, furent tapissés respectivement du tombeau de Mazarin (venu de la salle Coysevox), de celui de Jacques-Auguste de Thou, du Monument du Pont-au-Change (venu de la salle des Anguier) et du grand relief d'Alexandre et Diogène de Puget (fig. 4).

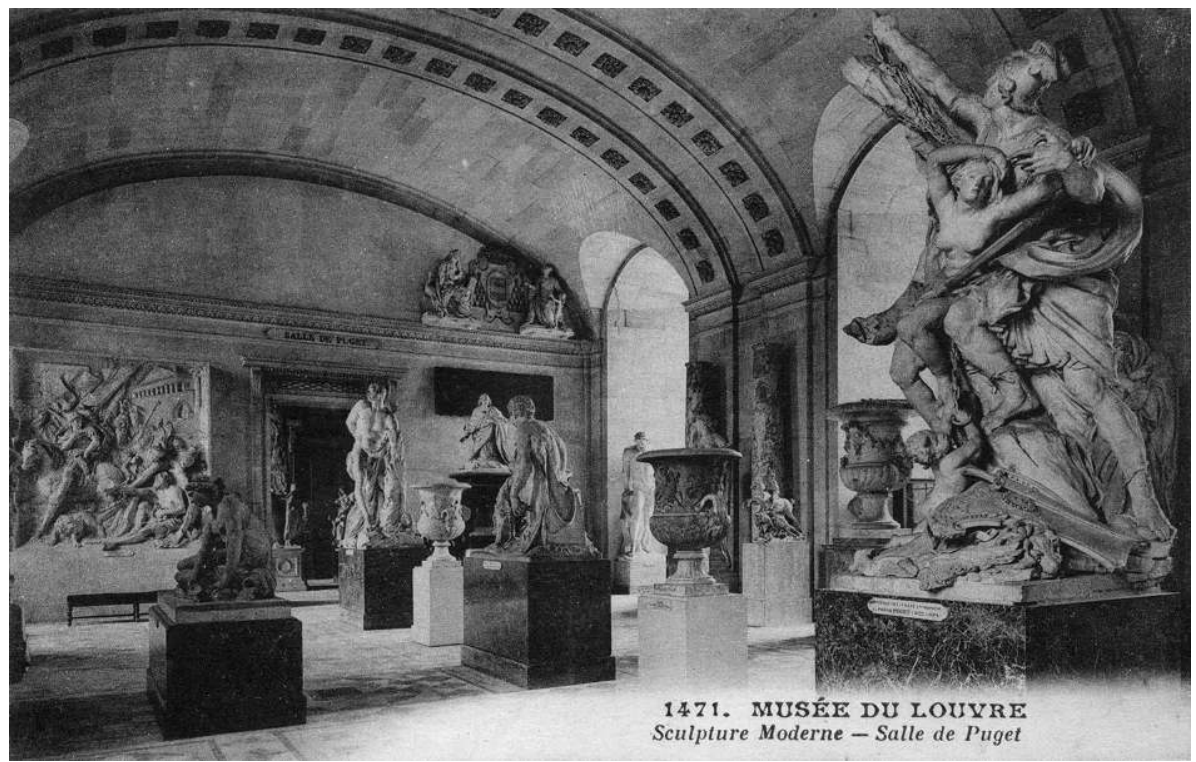

Dans les salles des Coustou et de Houdon, Courajod n'intervint qu'en densifiant la présentation par l'adjonction de bustes principalement

Bien entendu, la salle Rude fut abondamment garnie et transformée par les entrées successives de l'énorme fontaine de l'Observatoire de Carpeaux, en 1890, et du Napoléon séveillant à l'immortalité de Rude en 1892 (fig. 5 et 6). Il fallait encore grignoter un peu d'espace pour Carpeaux, mort en 1875, dont le Louvre attendait les œuvres avec impatience.

Fig. 5

La salle de Rude entre 1882 et 1897 Paris, musée du Louvre Documentation du département Documentation du département
des Sculptures, musée du Louvre des Sculptures, musée du Louvre
CDépartement des Sculptures du musée du Louvre

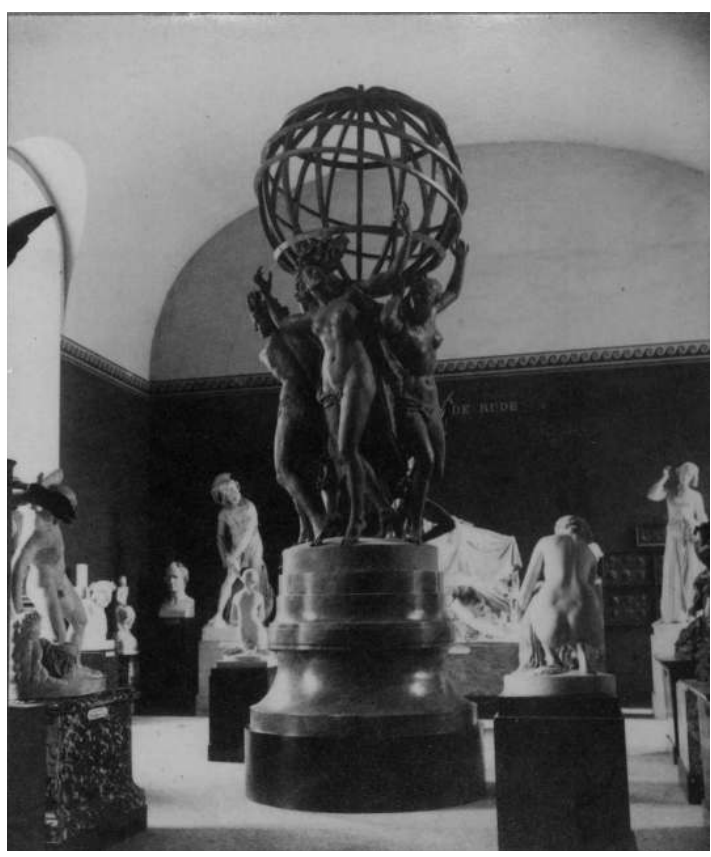

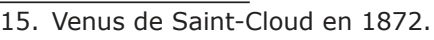

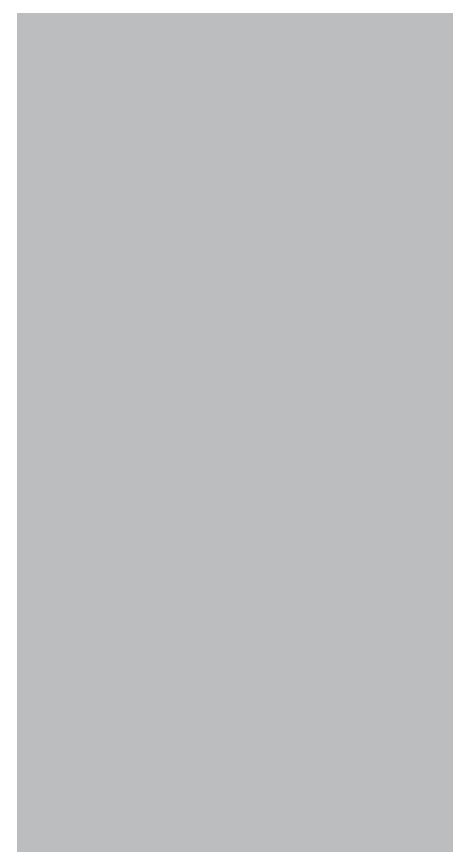




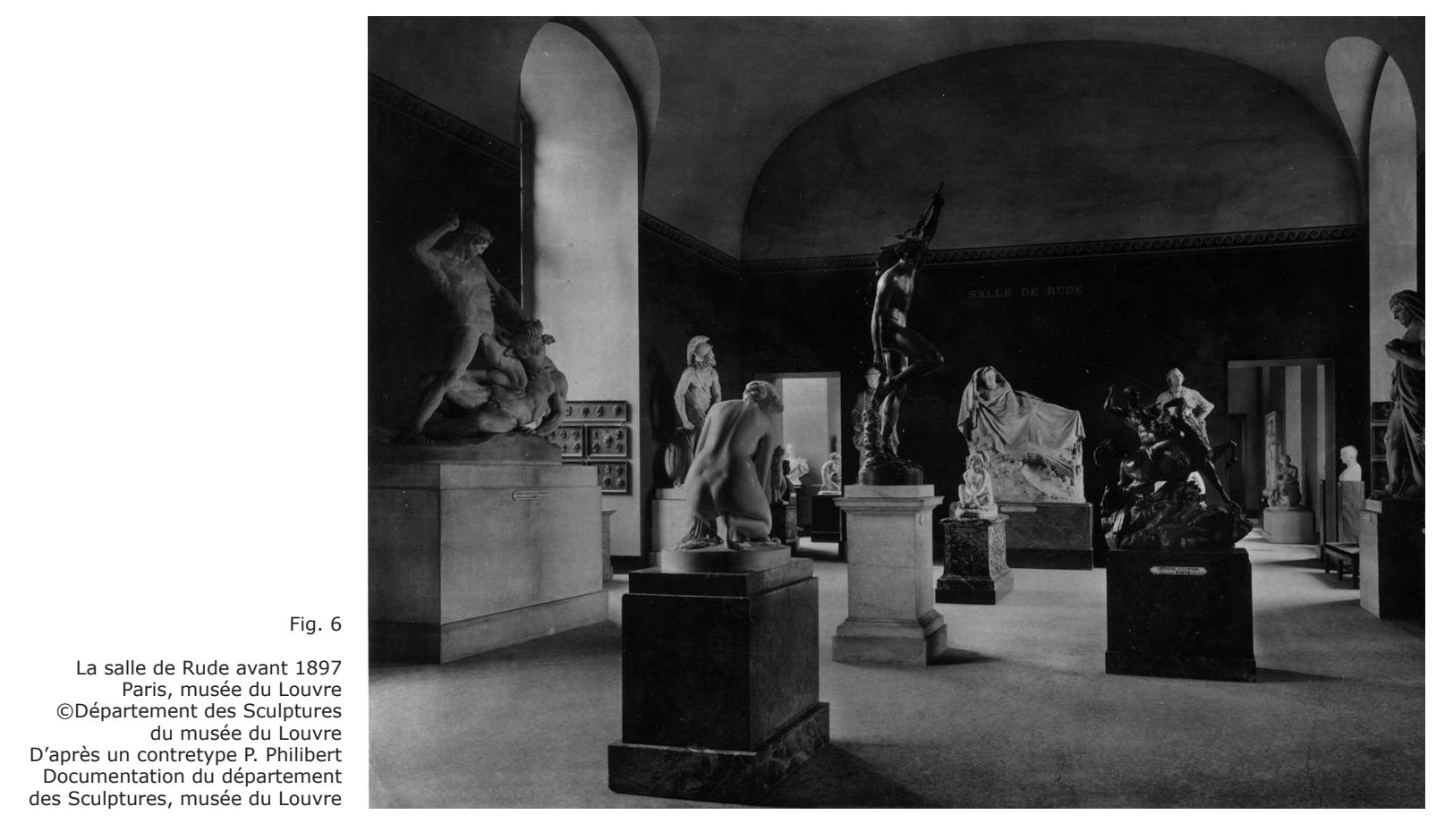

\section{André Michel et Paul Vitry (1896-1914): le retour au calme}

Le successeur de Courajod, André Michel, bien que l'ayant assisté pendant de 1893 à 1897 avait des conceptions plus sélectives, ainsi que son nouvel adjoint, Paul Vitry dont on ne peut pas séparer l'action ${ }^{16}$. Michel se plaignait de la pénurie de place et du manque d'éclairage harmonieux, plaidant pour des "salles alternées de dimensions inégales ", " avec des éclairages combinés, tantôt d'en haut, tantôt de côté ${ }^{17}$."

André Michel réussit à conquérir des espaces nouveaux et à assurer un parcours un peu plus cohérent au Sud, d'autant qu'on entrait désormais depuis les salles égyptiennes, à l'Est. Il ouvrit en 1908 une salle romane ${ }^{18}$, d'où avait été expulsée la cheminée de Bruges, et une autre consacrée au XIV siècle. D'ici on rejoignait la petite salle gothique et la salle Beauneveu. Michel restait fidèle à la centralité : la Vierge romane du Forez entre les colonnes de Coulomb pour l'art roman ; la croix de Saint-Léger-les-Troyes et trois statues de Vierge à l'Enfant pour la salle suivante, puisque le conservateur privilégiait dans ses acquisitions la statuaire mariale.

Pour les sculptures modernes, un grand espace fut gagné dans le prolongement de la salle de Rude, grâce au départ de la Chalcographie. Courajod y avait préparé une salle Carpeaux, ouverte par Michel en 1897, ornée évidemment d'un groupe central : le grand modèle de la fontaine de l'Observatoire en pivot. Michel ajouta en 1904 l'Ugolin rentré difficilement depuis le jardin des Tuileries, malgré les oppositions politiques, et la Flore pour faire pendant. Mais désormais, aucune œuvre n'était placée en second rang. Tout était sagement aligné le long des murs, sans trop d'accumulation.

Petit à petit, Michel réussit à grignoter le rez-de-chaussée de l'aile nord, jusqu'au guichet Marengo, pour exposer les sculpteurs du XIX ${ }^{e}$ siècle dans des salles aménagées par l'architecte Gaston Redon, le frère d'Odilon, puis par Victor Blavette après 1910. En 1908, il installa la salle Cavelier (qui dut s'effacer

16. Sur l'action d'André Michel dans les salles, G. Bresc-Bautier, « André Michel, conservateur et historien d'art. Défense et illustration de I'art français », Bulletin de la société de l'histoire de l'art français, 2012 (2013, en fait 2016), pp. 313-316.

17. André Michel, « Les accroissements du département des Sculptures », Gazette des BeauxArts, 3e période, t. avril 1912, p. 258

18. Auparavant Michel y avait exposé ses acquisitions récentes : les colonnes de Coulomb, la Vierge d'Abbeville, les statues provenant de Chantelle, une vitrine de maquettes et de petits sculptures. Guides Joanne, 1905, op. cit. note 1, p. 100, « Installation provisoire et souvent remaniée ». 
devant Eugène Guillaume après la guerre) et la salle Chapu de dimension réduite. On profita de l'occasion pour assurer un meilleur circuit, en ouvrant la porte qui donnait sur l'escalier Henri IV, en 1909, de manière à assurer une progression chronologique. Grâce à la donation Zoubaloff (1912), riche de bronzes et d'esquisses en plâtre de Barye, Michel put encore aménager deux salles qui buttaient sur le guichet Marengo : une salle Barye et un tout petit cabinet pour présenter les médaillons de David d'Angers. Elles échappaient au déroulé chronologique, puisque ces sculpteurs, très appréciés de Michel, l'un pour son naturalisme, l'autre pour son art du portrait, précédaient largement Rude, Carpeaux, Cavelier et Chapu. Ces petites salles, ouvertes en 1913, donnaient sur la rue de Rivoli, alors que se préparait une autre salle vers le Sud, consacrée à Dalou que Michel admirait particulièrement. Ce fut Paul Vitry qui l'ouvrit après la guerre, en 1921. Désormais, toute nouvelle extension était impossible. Après la guerre, les nouvelles arrivées du Luxembourg, en particulier les ouvres de Rodin, allèrent se glisser au milieu des peintures impressionnistes, avant que le grand projet - qui allait rester inachevé - de modernisation d'Henri Verne, vienne dans les années 1930, bouleverser la donne par le déménagement des collections de sculpture vers le pavillon des Sessions.

\section{L'auteur}

Archiviste paléographie et ancien membre de l'École française de Rome, Geneviève Bresc-Bautier s'est d'abord intéressée à l'art de la Sicile médiévale. Après avoir été conservateur au département des Sculptures du musée du Louvre depuis 1976, elle l'a dirigé de 2004 à 2014. Spécialiste de la sculpture française des XVI ${ }^{\mathrm{e}}$ et XVII ${ }^{\mathrm{e}}$ siècles, elle a été aussi chargée de la section consacrée à l'histoire du Louvre depuis 1989. Elle a écrit dans ce domaine plusieurs articles et livres et dirige actuellement un ouvrage collectif qui fera la synthèse en trois volumes de l'histoire du palais et du musée.

\section{The author}

Palaeographic archivist and former member of the École française de Rome was initially interested in the art of medieval Sicily. After having been a curator at the Department of Sculpture at the Musée du Louvre since 1976, she directed it from 2004 to 2014. Specialist in sixteenth- and seventeenth-century French sculpture, she has also been responsible for the section dedicated to the history of the Louvre since 1989. She has written several articles and books in this field and in currently editing a multi-author work in three volumes of the history of the palace and the museum. 